\title{
Exploring the Politeness of Instructional Strategies from Human-Human Online Tutoring Dialogues
}

\author{
JIONGHAO LIN, Centre for Learning Analytics, Monash University, Australia \\ MLADEN RAKOVIĆ, Centre for Learning Analytics, Monash University, Australia \\ DAVID LANG, Graduate School of Education, Stanford University, United States \\ DRAGAN GAŠEVIĆ, Centre for Learning Analytics, Monash University, Australia \\ GUANLIANG CHEN*, Centre for Learning Analytics, Monash University, Australia
}

\begin{abstract}
Existing research indicates that students prefer to work with tutors who express politely in online human-human tutoring, but excessive polite expressions might lower tutoring efficacy. However, there is a shortage of understanding about the use of politeness in online tutoring and the extent to which the politeness of instructional strategies can contribute to students' achievement. To address these gaps, we conducted a study on a large-scale dataset (5,165 students and 116 qualified tutors in 18,203 online tutoring sessions) of both effective and ineffective human-human online tutorial dialogues. The study made use of a well-known dialogue act coding scheme to identify instructional strategies, relied on the linguistic politeness theory to analyse the politeness levels of the tutors' instructional strategies, and utilised Gradient Tree Boosting to evaluate the predictive power of these politeness levels in revealing students' problem-solving performance. The results demonstrated that human tutors used both polite and non-polite expressions in the instructional strategies. Tutors were inclined to express politely in the strategy of providing positive feedback but less politely while providing negative feedback and asking questions to evaluate students' understanding. Compared to the students with prior progress, tutors provided more polite open questions to the students without prior progress but less polite corrective feedback. Importantly, we showed that, compared to previous research, the accuracy of predicting student problem-solving performance can be improved by incorporating politeness levels of instructional strategies with other documented predictors (e.g., the sentiment of the utterances).
\end{abstract}

CCS Concepts: • Applied computing $\rightarrow$ Education; E-learning; Distance learning.

Additional Key Words and Phrases: Learning Analytics, Educational Dialogue Analysis, Politeness, Student Performance, Prediction

\section{INTRODUCTION}

Tutoring is an effective form of instruction that can support students in different learning tasks across all educational levels [4, 35, 37]. The tutoring process often involves many instructional activities employed by tutors to guide student learning (e.g., providing corrective feedback and hints) and these activities can be conceptualised as instructional strategies, i.e., the approaches and principles employed by instructors to bring students towards the instructional goals $[13,34]$. Instructional strategies can be manifested as tutors' words or sentences [35, 37]. There are many types of instructional strategies including hints (e.g., "You may consider using the second condition"), open questions (e.g., "Why do you use this formula?"), and corrective feedback (e.g., "Yes, that is right" and "No, the second step is not correct") [8]. However, some strategies (e.g., directive hints and negative feedback) should be carefully used by tutors because these strategies might impose negative feelings on their students [7, 8], which might further impede students' motivation and acceptance of feedback [31]. For example, tutors can trigger students' feelings of restricted autonomy (e.g., by requesting students to take certain steps, "Solve the value of $x$ first") or neglect to show appreciation to students' efforts (e.g., by providing explicit negative feedback on students' work, "You're wrong. Try it again") [8]. These expressions that can harm students' self-efficacy are called direct expressions [6].

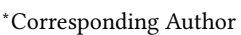


To mitigate the negative feelings that direct expressions can impose on students, human tutors often express their words in a polite manner (e.g., "Let us work together to solve the problem"). In this way, the tutors demonstrate solidarity and build rapport with students in the tutoring process [7,32], which has been demonstrated to benefit students' learning performance [23-26, 38-40]. Several previous studies highlighted the benefits of polite expressions in the tutoring practice $[24,26,36,38,39]$ and pointed out the necessity of including those polite expressions in the dialogue-based intelligent tutoring system (i.e., a computer-based tutoring system that guides students in a learning process, via natural conversation) [19,22]. However, tutors' excessive use of polite utterances in tutoring dialogue can have negative effects on learning process $[7,8,30]$. For instance, some human tutors might spend too much time working on polite expressions which can in turn hinder the tutoring process $[7,8,30]$. Moreover, the previous research demonstrated that students with high prior knowledge often prefer to receive instruction directly expressed [24].

Therefore, both benefits and hindrances of guiding students politely coexist in instructional communication. To maintain the effectiveness of one-on-one online tutoring, it is worth investigating the extent to which tutors express politeness in different instructional strategies (e.g., providing hints and corrective feedback). A promising way to investigate how tutors effectively communicate with students is to analyse instructional strategies based on student performance (e.g., whether students successfully completed learning tasks) [35]. Consequently, the appropriateness of expressing politely in the instructional communication can be reflected by investigating the relationship between the use of politeness in instructional strategies and student performance. However, few studies have examined this relationship in online one-on-one tutoring. As we posited the use of appropriate politeness can better facilitate the online one-on-one tutoring process, it is worth investigating this relationship to enhance the effectiveness of the instructional communication and further support students towards their learning goals.

To this end, our study aimed to analyse online human-human tutorial dialogues to shed light on the use of politeness in different instructional strategies. Specifically, we investigated different types of instructional strategies and their politeness levels in relation to the student prior progress (i.e., the progress on the problem that students made before joining a tutoring session) and the students' problem-solving performance. The study also aimed to examine the extent to which the politeness levels of instructional strategies can be used to predict students' problem-solving performance. Formally, our study was guided by the following two research questions:

- RQ 1 To what extent do tutors express politeness in different instructional strategies in online human-human tutoring?

- RQ 2 To what extent does the politeness level of instructional strategies predict students' performance in online human-human tutoring?

To answer RQ1, we firstly employed a widely-used dialogue act scheme (proposed by Vail and Boyer [37]) to identify tutor actions behind the utterances in a tutorial dialogue (e.g., asking a question and providing hints); these tutor actions were represented as instructional strategies. Then, we relied on the linguistic theories of politeness proposed by Brown and Levinson [6] to analyse the politeness levels in the tutors' utterances in online one-on-one tutoring. Building upon dialogue actions and politeness levels identified through the analysis of tutors' utterances, we investigated the politeness levels used in different instructional strategies. For RQ2, the politeness levels of instructional strategies were further used as input for training a well-established machine learning model -Gradient Tree Boosting (GTB) [9] -to predict student performance based on the statistical features (i.e., mean, standard deviation and median) of the politeness levels of instructional strategies in each tutoring session. 
Our results revealed that human tutors used both polite and direct expressions in the instructional strategies. Most tutors were inclined to express directly in comprehensive-gauging questions (e.g., "Does that make sense?") and negative feedback (e.g., "No, it is incorrect") and politely in positive feedback (e.g., "Well done, your result is correct"). Compared to the students who had made some prior progress on the problem before the start of a tutoring session, tutors were inclined to show more polite expressions in open questions but less polite in corrective feedback (i.e., negative feedback and positive feedback) to the students without prior progress. Lastly, by incorporating the politeness levels of instructional strategies with other documented factors (e.g., sentiment, time on task, and task complexity) [21], the GTB model achieved better performance of predicting student problem-solving performance compared to our previous work [21].

\section{RELATED WORK}

\subsection{Facework in Instructional Communication}

The central concept of Brown and Levinson's politeness theory is Face, which is the feeling of being respected by others [6]. In instructional communication, students' face can inevitably be threatened by tutors' utterances since tutors often restrict students' autonomy (e.g., direct students how to proceed to next step, "Solve the value of $x$ first") and may neglect students' need of appreciation (e.g, negative feedback, "No, it's wrong”) [8]. These face threatening utterances are called face-threatening acts (FTAs) [6]. If the tutors do not redress the FTAs in the instructional communication, tutors will instruct the students through direct expressions. In contrast, tutors can also express politely to mitigate FTAs to maintain students' face. For example, instead of directly requesting students to proceed to the next step (e.g., "Solve the value of $x$ first"), tutors can use more polite guidance (e.g., "Shall we work on the value of $x$ first?") to alleviate the feeling of restricting the autonomy.

\subsection{Effectiveness of Politeness in Instructional Communication}

Existing research suggests that polite tutors are more effective than direct tutors in guiding students' problem solving and to learn new concepts in different educational contexts [23-26, 38-40]. For example, Wang et al. [39] found that students guided by polite instruction (e.g., "Do you want to save the value now") had better performance than those guide by direct instruction (e.g., "Save the value now") in solving industrial engineering problem. The effectiveness of polite instruction on students' performance was further confirmed in learning foreign language [38]. In line with the findings presented in [39], McLaren et al. [24] also examined the effects of politeness in a web-based tutoring system and found that students who received polite tutorial instructions performed better than those who received direct tutorial instructions. Schneider et al. [36] showed that students who were given politely written task instructions outperformed their peers who were given direct instructions. Recently, Mikheeva et al. [26] found that the politely written feedback improved student performance in solving complex mathematical problems at the university level.

Despite many documented benefits of polite expression in previous work, tutors should avoid excessively expressing politeness in instructional communication $[7,8,18,30]$. The reasons can be summarised from two perspectives. First, excessive polite expressions might lead to negative effects on instructional efficiency. The earliest research of politeness in instructional communication pointed out that tutors should avoid rephrasing certain instructions completely in a polite expression as it might hinder the tutors' ability to give adequately informative feedback to students [30]. This claim was further confirmed by Brummernhenrich and Jucks [7]. They found that compared with the online tutoring sessions guided by polite tutors, non-polite tutors provided more hints, requests and answers to students' questions [7]. Second, the positive effect of politeness was conditioned on the levels of students' prior knowledge. Several empirical studies found that polite expression was only effective in supporting the students who had low prior knowledge (e.g., 
mastery levels on a learning subject) $[24,38,39]$. It should be noted that the students with high prior knowledge might feel more difficult to work with polite tutors than direct tutors on problem-solving tasks [24].

To make the online tutoring sessions effective, tutors should make a trade-off between instructing politely and directly [8]. Exploring the appropriate use of politeness in instructional expressions is therefore critical for tutors to maintain the productive communication. However, it is still unclear the extent to which tutors express appropriate politeness in different instructional strategies in online one-on-one tutoring. The studies by $[7,8]$ concluded that there was a clear connection between politeness and instructional strategies but they did not reveal how the tutors strike the balance between the direct and polite expressions in the tutoring process. The study presented in this paper, instead, investigated the extent to which tutors expressed politeness in instructional communication on a large scale tutorial dialogue dataset. Secondly, the studies by [7, 8] did not investigate the changes of the politeness levels of instructional strategies as the tutoring sessions progressed. In contrast, our previous work found that in comparison to ineffective tutoring sessions, in effective tutoring sessions where students successfully collaborated with tutors to solve problems, tutors tended to use polite expressions at the start of the online tutoring sessions and gradually used more direct expressions to instruct students as the tutoring sessions progressed [19]. However, it is still unclear whether the effectiveness of tutoring associated with the politeness levels of instructional communication as the tutoring progressed.

\section{METHODS}

\subsection{Dataset}

We analysed the tutor-student dialogue dataset provided by an online educational company. Our research was approved by the Human Research Ethics Committee of Monash University under Project ID 26156. The dataset contained data about 5,165 K-12 students and 116 qualified tutors. The students and tutors were working collaboratively on solving problems in different STEM subjects such as mathematics, chemistry and physics. A tutoring session was commonly initiated by a student by sharing unsolved problems and an experienced tutor was then allocated to work collaboratively with the student, rather than to directly provide students with a simple solution. Therefore, the communication between tutors and students contained fine-grained details about the scaffolding process in solving problems. The dataset originally contained 18,203 dialogue sessions but some short tutoring sessions did not have sufficient tutoring guidance. Therefore, we discarded the dialogue sessions that concluded within the first minute and contained less than 10 utterances. We also discarded the sessions where the students' prior progress was not available and the details of the process followed to determine the students' prior progress is described in Sec. 3.2. After removal, the final dataset contained 14,562 tutorial sessions and $92 \%$ of them were related to the maths tutoring.

\subsection{Data Pre-processing}

Yang and $\mathrm{Li}$ [42] suggested that tutors should select appropriate instructional strategies with the consideration of the students' prior progress which was positively associated with students' self-efficacy and problem-solving performance. In the stage of data preparation, we first manually annotated the students' prior progress based on the tutor-student utterances. To this end, we identified students' prior progress in each session by observing the first several utterances from tutors and students. At the start of a tutorial session, tutors often asked students about the progress that they made before joining the tutoring sessions. For instance, a tutor might ask "What you have done on this problem?", and a student might answer "Nothing" or "Yes, let me show you" and then described the progress they had made. By observing the first few tutor-student utterances of a tutorial dialogue, we annotated the tutoring sessions as either With-Prior-Progress or 
Table 1. The descriptive statistics of the dataset. WP and WoP denote With Prior Progress and Without Prior Progress, respectively. Mann-Whitney tests were applied to examine differences (Rows 5-9) between any pair of the Gap-clarified, Gap-explained, and Gap-bridged categories in which students had the same level of prior progress. All differences were significant $(p<0.01)$.

\begin{tabular}{|c|c|c|c|c|c|c|c|}
\hline $2 *$ Metric & $2^{*}$ All & \multicolumn{2}{|c|}{ Gap-clarified } & \multicolumn{2}{|c|}{ Gap-explained } & \multicolumn{2}{|c|}{ Gap-bridged } \\
\hline 1. \# total sessions: & 14,562 & 1,203 & 1,302 & 1,255 & 1,931 & 4,482 & 4,389 \\
\hline 2. \# total uttrances: & $1,216,784$ & 31,014 & 30,128 & 78,575 & 113,099 & 475,849 & 488,119 \\
\hline 3. \# tutors: & 116 & 92 & 96 & 98 & 99 & 110 & 106 \\
\hline 4. \# students: & 5,165 & 763 & 962 & 908 & 1,419 & 1,800 & 2,168 \\
\hline 5. Avg. Sess Dur (mins): & $30.27 \pm 30.66$ & $10.55 \pm 7.64$ & $9.75 \pm 7.21$ & $25.94 \pm 19.03$ & $22.88 \pm 18.05$ & $37.78 \pm 32.17$ & $38.60 \pm 37.37$ \\
\hline 6. Avg. \# Uttr / Sess: & $83.56 \pm 81.05$ & $25.78 \pm 16.68$ & $23.14 \pm 14.92$ & $62.61 \pm 43.79$ & $58.57 \pm 42.73$ & $106.17 \pm 87.62$ & $111.21 \pm 93.70$ \\
\hline 7. Avg. \# Words / Sess: & $647.75 \pm 596.12$ & $201.62 \pm 131.81$ & $198.13 \pm 134.44$ & $524.09 \pm 351.18$ & $489.56 \pm 346.82$ & $807.46 \pm 649.08$ & $845.28 \pm 675.05$ \\
\hline 8. Avg. \% Uttr by tutors: & $58.42 \pm 7.86$ & $53.95 \pm 9.49$ & $56.46 \pm 9.51$ & $58.68 \pm 7.82$ & $60.25 \pm 7.77$ & $58.03 \pm 7.07$ & $59.75 \pm 6.94$ \\
\hline 9. Avg. \% Words by tutors: & $78.36 \pm 9.10$ & $74.32 \pm 11.80$ & $80.54 \pm 10.20$ & $78.87 \pm 8.59$ & $82.21 \pm 7.96$ & $76.09 \pm 8.69$ & $79.30 \pm 7.81$ \\
\hline
\end{tabular}

Without-Prior-Progress. The annotation process was first implemented by two human coders. Each dialogue was labelled by two coders independently. The percentage of agreement between two human coders was 0.847 . The Cohen's $\kappa$ score of 0.735 was derived as a measure of inter-rater reliability of the data labelling, which demonstrated a substantial level of agreement. The disagreement between the two coders was resolved by a third independent coder. It should be noted that we had no access to the information indicating students' prior knowledge on the mastery levels of a specific learning subject (e.g., mathematics). Instead, we included the student prior progress (i.e., the progress that students made on the consulted problem before joining a tutoring session) as the proxy of student prior knowledge, which was in line with the definition of student prior knowledge (i.e., student experiences on the learning contents before they having a tutoring session [14]).

\subsection{Descriptive Statistics of the Dataset}

To answer research questions RQ1 \& 2, we identified the students' performance for each tutoring session since incorporating the students' performance in the analysis can better inform the appropriateness of using politeness in instructional strategies. To this end, we annotated each tutorial session based on the student's performance at the end of a session. Instead of annotating each session as effective and ineffective (e.g., [35, 37]), our study provided a more fine-grained approach to distinguish the performance level of a student, which was listed in below three categories:

- Gap-clarified: a tutor identified the problem but was unsure whether the student made any progress at the end of the tutoring session;

- Gap-explained: a tutor identified the problem and assisted the student to make certain progress, but the student did not completely solve the problem;

- Gap-bridged: a tutor identified the problem and guided the student to successfully solve the problem.

Each tutorial dialogue was labelled by an educational expert employed by the data provider (i.e., the educational technology company). To validate the reliability of their annotations, we randomly selected 500 tutorial dialogues from the whole dataset and annotated them independently by using the same coding rules. The result of validation reached Cohen's $\kappa$ score of 0.787 . We provided sample dialogues for each of the above three categories in an electronic appendix, which is accessible via https://bit.ly/lak22_tutor_appendix.

The descriptive statistics of the dataset are shown in Table 1 . We observed that most of the dialogues were of the Gap-bridged sessions (8,871, 60.9\%), followed by Gap-explained (3,186, 21.9\%), and then Gap-clarified (2,505, 17.2\%). This indicated that more than half of the students solved their problems at the end of the sessions. Additionally, we observed that more than $47 \%$ of the students made some progress on the problem before joining a tutoring session 
Table 2. The description of the instruction strategies derived from Brummernhenrich and Jucks [8] and Lin et al. [21]

\begin{tabular}{lll}
\hline Instructional Strategy in [8] & Instructional Strategy in Our Work [21] & Sample Utterances \\
\hline $2^{*}$ Hints & $\begin{array}{l}\text { Information Hint } \\
\text { Observation Hint }\end{array}$ & $\begin{array}{l}\text { "It can be any one of the cards in the deck." } \\
\text { "We have 80." }\end{array}$ \\
\hline Closed question (prompt) & Probing Question & "How many options can it be?" \\
\hline Open-ended Questions & Open Question & "What do you think we could try next?" \\
\hline Comprehension-gauging questions & Evaluation Question & "Does that make sense?" \\
\hline Explanation & Explanation & "The straight line is the line on the bottom." \\
\hline $2^{*}$ Positive Feedback & General Positive Feedback & "Correct!" \\
\hline Negative Feedback & Elaborated Positive Feedback & "Your formula for period is correct!" \\
\hline
\end{tabular}

Table 1. Rows 5-7 of Table 1 showed that tutors and students invested more effort in the Gap-bridged sessions than the other two. An explanation for this might be the more efforts tutors and students invested in tutorial sessions, the better problem-solving performance achieved by student.

\subsection{Inferring the Instructional Strategies from Instructional Communication}

To answer RQ1, we identified the instructional strategies as shown in the tutors' utterances. We drew from the work by Brummernhenrich and Jucks [8], who investigated the politeness in seven types of commonly used instructional strategies, which were presented in the first column of Table 2. Brummernhenrich and Jucks [8] identified the instructional strategies by relying on the efforts of experienced human coders, which was quite time-consuming and cost-demanding to identify the instructional strategies on a large amount of tutors' utterances. Instead, in our study, we automated the process of identifying the instructional strategies. We characterised instructional strategies by capturing the actions (i.e., dialogue acts) hidden behind tutors' utterances; this was informed by prior research [27, 35, 37]. To automatically identify the dialogue acts, our previous work [21] applied the BERT language model [1] to train a dialogue act classifier, which was built on 3,629 utterances (training, validation, and testing datasets in the ratio of 80\%:10\%:10\%) from tutors and students. It should be noted that the dialogue acts classifier was trained on the same dataset as the current study. After tuning the classifier with the optimal hyper-parameters, the classifier achieved sufficient performance (F1 score 0.742 and Cohen's $\kappa$ score of 0.735 ) on identifying 31 dialogue acts (composed of non-instructional and instructional communication) from tutors and students, which were built upon the well-known dialogue acts scheme [37]. For the current study, we only focused on the tutors' dialogue acts which matched instructional strategies proposed in [8]. Compared with the the work by Brummernhenrich and Jucks [8], our identified instructional strategies presented a more fine-grained level (as shown in the second column of Table 2). For instance, the strategy Hint was differentiated as Information hint (i.e., prompt students work on the next step) and the Observation hint (i.e., remind students of known information).

\subsection{Inferring the Politeness from Instructional Communication}

A tutor's utterance could be interpreted as a certain type of instructional strategy and be reflective of a certain level of politeness. The recognition of politeness levels could further help researchers understand the extent to which human tutors expressed politeness in instructional strategies. To this end, we employed the well-established politeness level scoring tool (available in the Github repository ${ }^{1}$ ) by Niu and Bansal [29] to quantify the politeness levels of tutors' utterances. This tool was originally developed to classify textual data into direct or polite categories. In addition to

\footnotetext{
$\overline{{ }^{1} \text { https://github.com/WolfNiu/polite-dialogue-generation }}$
} 
assigning the polite or direct categories, this tool also generated a score to indicate the politeness level from 0 (most direct) to 1 (most polite) [29]. The generated politeness scores, hence, helped us understand the politeness levels for tutors' utterances in the instructional communication. Since this tool was originally used to classify the textual data as either polite or direct, we validated the reliability of this tool on our dialogue dataset as follows. We recruited two human coders to independently annotate 500 utterances (randomly sampled from our dialogue data) into polite and direct labels. The percentage of agreements between two human coders was 0.724 and the Cohen's kappa score was 0.531, which was enough for analysis [28]. The inconsistency between two human coders was then resolved by the third human coder. Then, we used our annotated results to compare the classified results by the tool [29] and obtained Cohen's $\mathrm{k}$ score of 0.639 , which presented a sufficient score to use this tool for analysis.

To infer the politeness levels of instructional strategies, we first used the dialogue act classifier developed in our previous work [21] to identify the hidden instructional strategies from tutors' utterances. Then, we used the politeness level scoring tool by [29] to identify the politeness levels of the same tutors' utterances. Finally, we paired the instructional strategies and the politeness levels based on the same tutors' utterances to represent the politeness levels of instructional strategies.

\subsection{Predicting Student Performance}

3.6.1 Prediction Model. To answer RQ2, we trained a Gradient Tree Boosting (GTB) [9] machine learning model to predict the student performance (i.e., Gap-clarified, Gap-explained, and Gap-bridged) and a Random Forest model as the comparison on the prediction. The Random Forest model was built upon the idea of the ensemble method [12] which claimed that combining many predictors to make predictions on the same task can obtain better performance than an individual predictor. [15] found that the Random Forest model had generally better performance compared to 179 commonly used machine learning models (e.g., Naive Bayes, Decision Trees, and Support Vector Machines) on 121 different classification tasks. Therefore, our study adopted Random Forest classifier to answer RQ2. Similar to the Random Forest model, GTB also processed the data by a collection of decision tree models as the predictors, and the final prediction was made by considering the predictions from all constructed predictors. It should be noted that each decision tree predictor was trained on a sample randomly selected from the data so each decision tree was distinct from others. These distinct decision trees work collaboratively to capture patterns in the data to make predictions. The main difference between GTB and Random Forest was the collaborative approach of predictors. For Random Forest, the predictors were constructed independently and many of them could produce the same type of prediction error. In contrast, the predictors in GTB were constructed sequentially so that the prediction errors made by previous predictors can be corrected by the subsequent predictors. We selected GTB model to answer research RQ2 due to its accuracy in our previous study [21] in predicting student performance on the same dataset.

3.6.2 Feature Engineering. To train the GTB classifier, we engineered a total of 27 statistical features (i.e., mean, standard deviation, and median suggested by [41]) about strategy politeness, which included: (i) average politeness level of the nine instructional strategies used in our study as shown in Table 2; (ii) standard deviation of politeness level of nine instructional strategies; and iii) median values of politeness level of nine instructional strategies. We denoted these features as Strategy-politeness Feat. We assumed that students' prior progress on a problem solving task might be beneficial in enhancing the model performance. However, we did not include the students' prior progress into the feature set because we aimed to build a predictive model for real-time use (i.e., the input features to the model should be automatically generated from the observed data) and the progress level was not readily available. 
The current study mainly focused on the politeness features of instructional strategies produced by tutors. However, as student performance could be influenced by other factors (e.g., student sentiment [17]) in the tutoring process, we also included those non-politeness features in our final model. Building upon our previous work [21], we engineered additional 543 non-politeness features to enhance our feature set, which included:

- Effort, which measured three activities from tutor-student utterances in a tutoring session (i.e., the duration of the tutorial session, the number of utterances, and the total number of words in utterances).

- Informativeness, which counted the number of concepts and unique words in the tutor and student utterances.

- Complexity, which calculated the Flesch readability score [11] from the utterances tutors and students.

- Responsiveness, which measured the average time that students spent waiting for the tutor's reply after the request for help had been made.

- \# Questions, which counted the number of questions that tutors and students created.

- Entrainment, which measured the level of lexical consistency [5] that a tutor's and student's utterances reached in a tutoring session.

- Sentiment, which described the sentiment levels, ranging from -1 (most negative sentiment) to +1 (most positive sentiment), detected in utterances from tutors/students. The sentiment levels were generated by the VADER model [16].

- Experience, which described the number of tutorial sessions that students and tutors participated in before the current one.

- Dialogue Acts, which described the intention behind the tutors/students' utterances (e.g., asking questioning). This group of features was composed by the number of specific dialogue acts made by students and tutors in a dialogue, the fraction of specific dialogue acts made by students and tutors in a dialogue, and the number of sequential dialogue act patterns made by students and tutors in a tutoring session.

- $\mathbf{N}$-grams, which contained the top 100 most frequent unigrams, bigrams, and trigrams lexicons extracted from the tutor-student utterances.

3.6.3 Study Setup. Our study evaluated the prediction performance of the GTB model by comparing with the RF model (baseline model). Before training both models, we randomly allocated the full annotated dataset into training set, validation set, and testing set with the proportion of $80 \%: 10 \%: 10 \%$, respectively. Then, both models were trained using the Python package scikit-learn ${ }^{2}$. The hyperparameters (e.g., the maximum depth of the tree) in both models were tuned by using grid search on the validation dataset. Finally, both models' performance was evaluated on the testing set. To evaluate models' performance, we employed four representative metrics, i.e., classification accuracy, F1-score, Area Under the Curve (AUC), and Cohen's $k$ score.

3.6.4 Ablation Test. To gain a better understanding of the predictive power of different types of features, we also measured the respective contributions made by all features (both strategy-politeness and non-politeness features) by conducting an ablation test, which is a widely-used method by researchers to assess contributions made by features on the performance of a prediction model $[20,21]$. The contribution of a feature was measured by calculating the difference between the prediction performance of a model including the feature and the model excluding the feature.

\footnotetext{
${ }^{2}$ https://scikit-learn.org/
} 


\section{RESULTS}

\subsection{Results on RQ1}

Table 3. The politeness level of instructional strategies sorted according to the values of their average politeness levels in the dataset in an ascending order, i.e., the column All. WP and WoP denote With Prior Progress and Without Prior Progress, respectively.MannWhitney test was applied to examine the difference between WP and WoP for each session categories (significant results were in bold font, $p<0.001$ ), and the difference between any two of the Gap-clarified, Gap-explained, and Gap-bridged categories in which students had the same level of prior progress (significant results were marked with the same symbol ${ }^{3}$ in a row, $p<0.001$ ).

\begin{tabular}{|c|c|c|c|c|c|c|c|c|}
\hline & $2^{*}$ Strategy & $2^{*} \mathrm{All}$ & Gap-c & arified & Gap-e & plained & Gap-l & idged \\
\hline & & & WP & WoP & WP & WoP & WP & WoP \\
\hline 1. & Evaluation Question & 0.22 & 0.24 & 0.22 & 0.22 & 0.22 & 0.22 & 0.22 \\
\hline 2. & Negative Feedback & 0.39 & 0.43 & $\diamond 0.42$ & 0.40 & $\diamond 0.37$ & 0.40 & 0.38 \\
\hline 3. & Probing Question & 0.44 & * 0.46 & +0.48 & $\dagger 0.45$ & $\dagger 0.46$ & $\dagger 20.43$ & $\dagger * 0.44$ \\
\hline 4. & Open Question & 0.49 & 0.52 & $\diamond+0.63$ & 0.48 & $\diamond 0.52$ & 0.47 & +0.49 \\
\hline 5. & Observation Hint & 0.56 & $* 0.60$ & $\diamond * 0.61$ & $\dagger 0.58$ & $\diamond \dagger 0.58$ & $\dagger+0.56$ & $\dagger * 0.56$ \\
\hline 6. & Explanation & 0.57 & * 0.61 & 0.60 & 0.58 & 0.57 & +0.57 & 0.57 \\
\hline 7. & Information Hint & 0.62 & $\diamond * 0.65$ & $\diamond * 0.65$ & $\diamond 0.62$ & $\diamond \dagger 0.63$ & 20.62 & $\dagger * 0.62$ \\
\hline 8. & Elaborated Positive Feedback & 0.63 & 0.64 & 0.65 & 0.65 & $\dagger 0.65$ & 0.63 & $\dagger 0.62$ \\
\hline 9. & General Positive Feedback & 0.72 & \& 0.74 & $\diamond * 0.75$ & $\dagger 0.73$ & $\diamond \dagger 0.74$ & $\dagger \div 0.72$ & $\dagger * 0.72$ \\
\hline
\end{tabular}

The average politeness levels of instructional strategies are presented in Table 3. These instructional strategies are sorted based on the values of their average politeness levels calculated in the whole dataset. The results indicated that, in real-world online one-on-one tutoring, tutors tended to express different politeness levels of instructional strategies in the tutoring process to guide students. The three most direct instructional strategies in our dataset were Evaluation Question (e.g., "Does that make sense?"), Negative Feedback (e.g., "No, it is incorect”), and Probing Question (e.g., "What if you add the value of $y$ ?"), and the three most polite ones were General Positive Feedback (e.g., "Correct”), Elaborated Positive Feedback (e.g., "Yes, you use the function correctly"), and Information Hints (e.g., "Hint: you might use the third condition").

We investigated the difference of politeness levels of the instructional strategies between any pair of the Gap-clarified, Gap-explained, and Gap-bridged sessions. A pair-wise comparison was conducted between students with and without prior progress and the differences were examined by the Mann-Whitney test; the significant results are marked with the same symbol (i.e., $\diamond, \dagger, *)$ in Table 3. We found that tutors in the Gap-bridged sessions presented lower average politeness levels of the strategies (Probing Question, Observation Hint, and General Positive Feedback) than in the other two categories of sessions (i.e., Gap-explained and Gap-clarified) in both groups of students with and without prior progress. This finding indicated that tutors in the most effective sessions (i.e., Gap-bridged) tended to use direct expressions of these instructional strategies to guide students. Then, we scrutinised the politeness levels of the instructional strategies between With-Prior-Progress and Without-Prior-Progress for each session category. We found that the politeness levels of the instructional strategies Negative Feedback and Elaborated Positive Feedback when used with the With-Prior-Progress students were statistically higher than those used with the Without-PriorProgress students in the Gap-bridged session categories. This result indicated that the tutors tended to give explicit and direct expressions of feedback to the Without-Prior-Progress students. Additionally, we also found that Without-PriorProgress students received more polite expression of Open Questions from tutors compared to their With-Prior-Progress counterparts in the Gap-bridged sessions.

\footnotetext{
${ }^{3}$ We used three symbols to mark the statistical differences. $\diamond$ marked the difference between Gap-clarified and Gap-explained, $\dagger$ marked the difference
} between Gap-explained and Gap-bridged, * marked the difference between Gap-clarified and Gap-bridged 


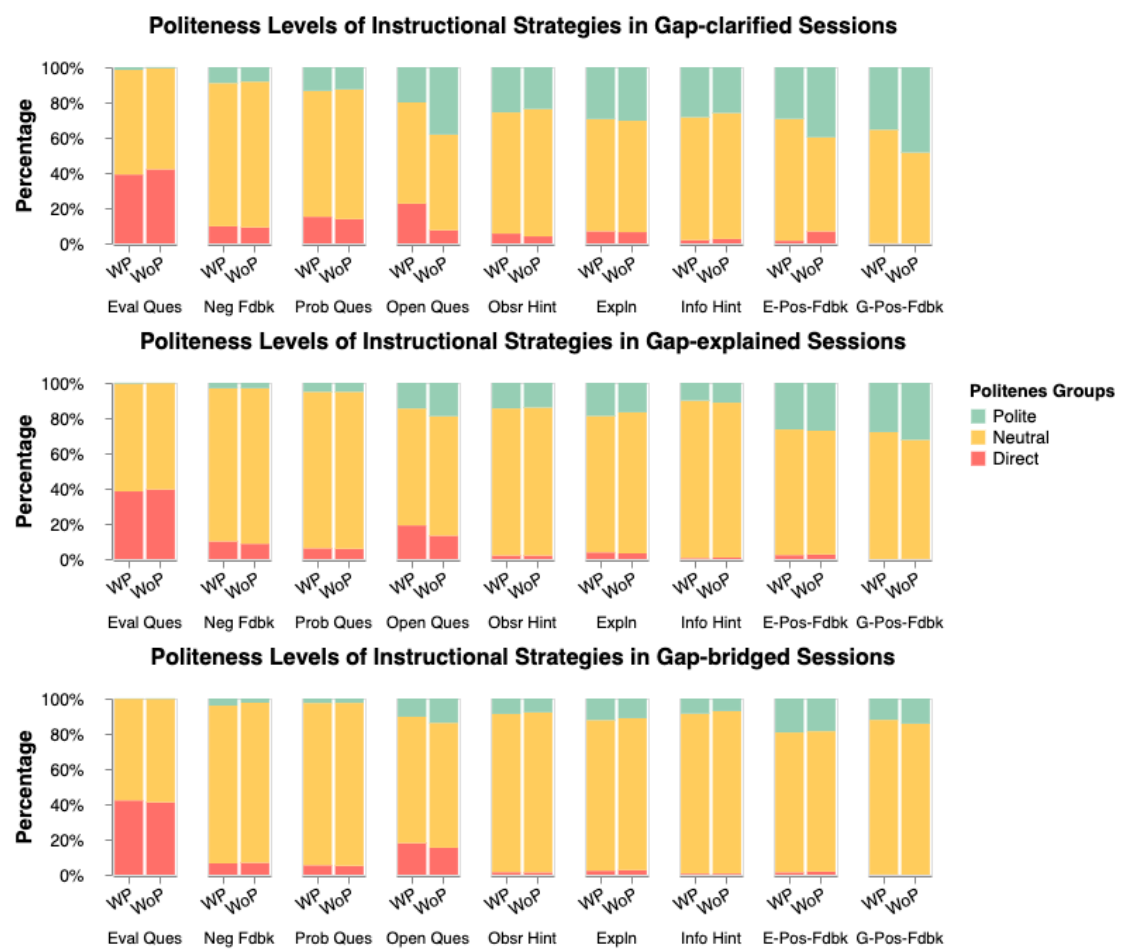

Fig. 1. The distribution of the politeness groups (i.e., Polite, Neutral, and Direct) for each instructional strategy in different session categories. WP and WoP denote With Prior Progress and Without Prior Progress, respectively. The abbreviations include Eval-Ques (Evaluation Question), Neg-Fdbk (Negative Feedback), Prob Ques (Probing Question), Open Ques (Open Question), Obsr Hint (Observation Hint), Expln (Explanation), Info Hint (Information Hint), E-Pos-Fdbk (Elaborated Positive Feedback), and G-Pos-Fdbk (General Positive Feedback).

We further investigated the distribution of politeness in instructional communication (shown in Fig. 1). Building upon the suggestions by Niu and Bansal [29], we categorised the politeness levels of tutors' utterances into three politeness groups (i.e., the politeness levels of tutors' utterances in the range $(0.8,1.0]$ as Polite, $[0.2,0.8]$ as Neutral, and $[0,0.2)$ as Direct). These politeness groups were used to demonstrate the variance of instructional strategies' politeness levels. Taking the strategy Evaluation Question (i.e., Eval Ques in Fig. 1) as an example, less than 5\% of the Evaluation Question strategies were presented in the polite form (e.g., "I hope this make sense?"), $40 \%$ in direct (e.g., "Do you understand what I mean?"), and more than $50 \%$ in neutral (e.g., "Would that make sense?"), which is shown for the three different types of sessions in Fig. 1). Additionally, the percentage of polite General Positive Feedback (e.g., "Brilliant!") in the Gap-bridged session was less than the other two sessions. Instead, tutors in Gap-bridged sessions used more neutral form (e.g., "Yes, that is right") to inform the correctness. It should be noted that tutors from three different sessions rarely used the direct form of General Positive Feedback (e.g., "Exactly as what I got").

Inspired by the variation of politeness existed within each instructional strategy, we further investigated the variation of instructional strategies' politeness as the tutorial progressed and in particular at the early stage of the tutoring which is important to build the rapport with students [3]. Based on our previous work [21], some instructional strategies (e.g., Negative Feedback and Evaluation Question) were not used frequently by tutors so the occurrence of these strategies was also less frequent compared with the commonly-used instructional strategies (e.g., General Positive 

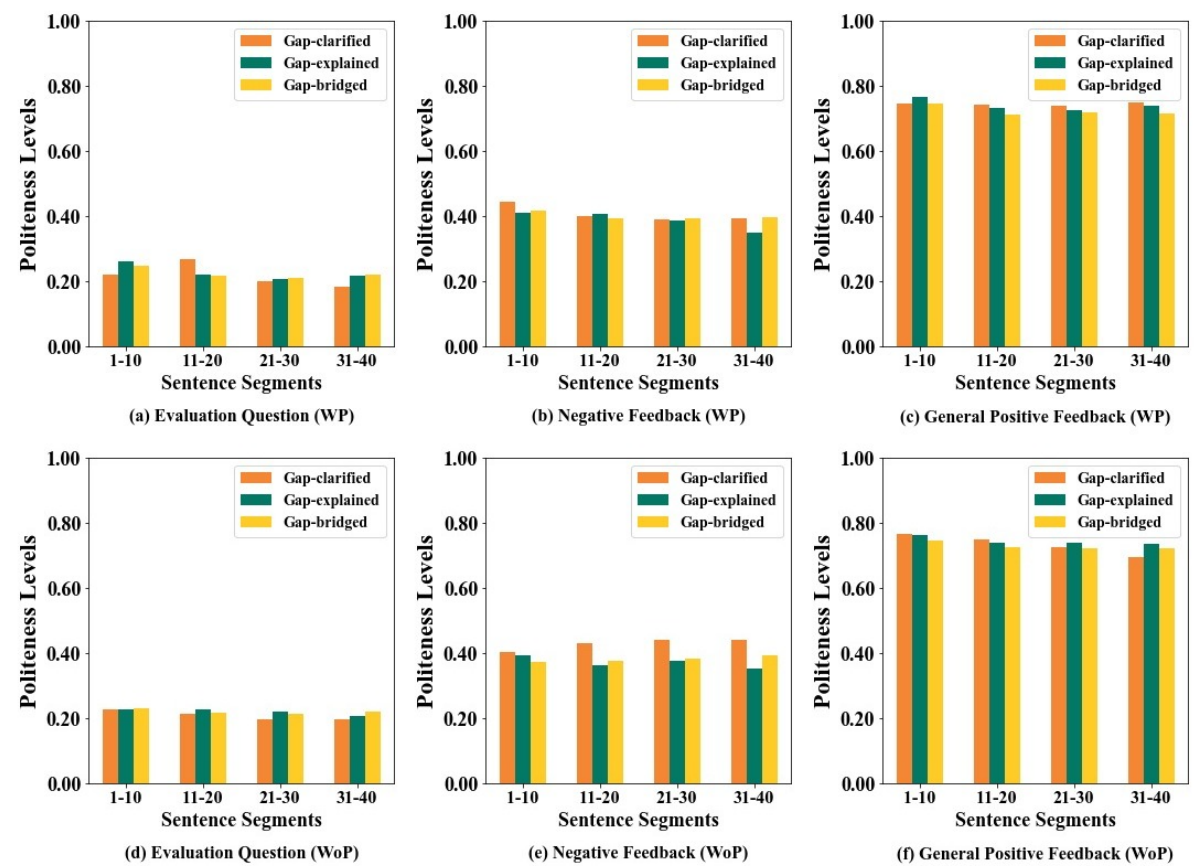

Fig. 2. The distribution of tutors' sentences associated with different average politeness levels of instructional strategies as tutorial sessions progressed. WP and WoP denote With Prior Progress and Without Prior Progress, respectively.

Feedback and Probing Question) as the tutoring progressed. To obtain less biased results, we computed the average politeness levels of every instructional strategy in the first [1, 10], [11,20], [21,30], and [31, 40] utterance segments for the three session categories. Due to the limited space, we only presented the results for the three instructional strategies (i.e., Evaluation Question, Negative Feedback, and General Positive Feedback) in Fig. 2. The results of other instructional strategies can be found in the digital appendix ${ }^{4}$. To examine the changes of strategies' politeness as tutoring progressed, Mann-Whitney U tests were applied to measure the difference (significant difference was measured by $p<0.001$ ) between the two consecutive segments in the same session category. In the sessions where students had prior progress, tutors in Gap-bridged and Gap-explained sessions asked more polite Evaluation Questions in the utterance segment $[1,10]$ than in $[11,20]$ (Fig. 2 (a)) but no difference between segments $[11,20]$ and $[21,30]$ and between segments $[21,30]$ and $[41,40]$ were found. In comparison, in the sessions where students had no prior progress (Fig. 2 (d)), we did not find significant differences in politeness levels of the Evaluation Question strategy between the consecutive utterance segments across the three session categories. When scrutinising the strategy Negative Feedback (Fig. 2 (b) and (c)), we did not observe significant differences in politeness levels between the consecutive utterance segments in the three session categories. Regarding the strategy General Positive Feedback in Fig. 2 (c) and (f), tutors in Gap-bridged and Gap-explained sessions delivered this strategy more politely in the utterance segment $[1,10]$ than in $[11,20]$ but no significant differences were found between segments $[11,20]$ and $[21,30]$ and between segments $[21,30]$ and $[41,40]$. 

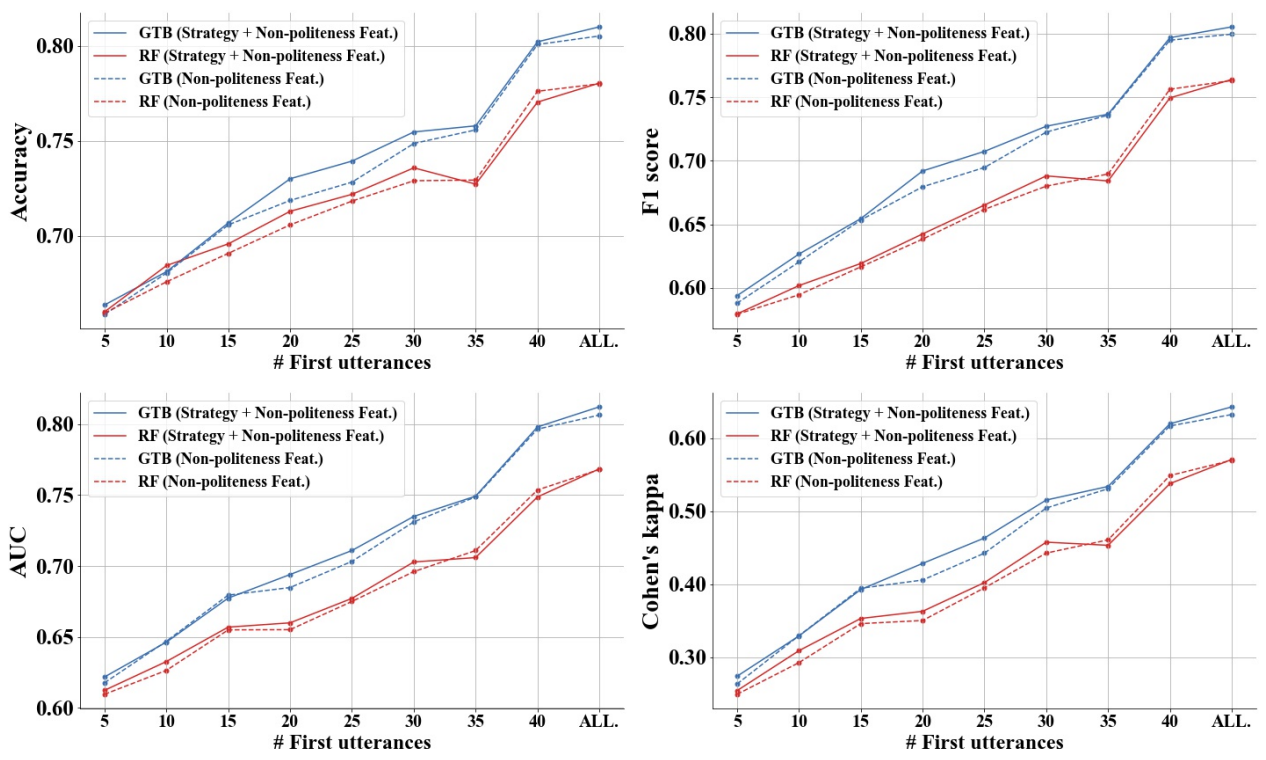

Fig. 3. The performance of GTB and Random Forest in predicting student performance in solving problems.

\subsection{Results on RQ2}

We answered RQ2 from two different perspectives. Firstly, we investigated the effectiveness of the feature set (i.e., strategy-politeness features and non-politeness features) designed in Sec. 3.6 in predicting student performance. To this end, we decided to use the first $\mathrm{N}$ utterances (where $\mathrm{N} \epsilon[5,10,15,25,30,35,40, A l l]$ utterances from the whole dialogues) to extract the features and those features were then used as the input for both Random Forest and GTB algorithms. As shown in Fig. 3 (solid lines), GTB outperformed Random Forest in predicting student performance when using all utterances from the tutoring sessions as the input and GTB achieved the performance of $0.810,0.805,0.812$, and 0.643 as measured by Accuracy, F1 score, AUC, and Cohen's $\kappa$, respectively. To further examine the power of strategy-politeness features in predicting students' performance, we used the non-politeness features as the comparison to train both algorithms. The results (dash lines in Fig. 3) indicated that the GTB model trained on non-politeness features achieved similar prediction performance at the earlier stage of the tutoring (i.e., $N=5,10,15$ ) compared to that of the model trained on both strategy-politeness features and non-politeness features. This might be the reason that tutors commonly used more non-instructional communication (e.g., Greeting) at the outset of the tutoring [27]. Some instructional strategies in tutoring sessions might not be frequently used at the outset of the tutoring such as evaluation question (e.g., "Does that make sense?”). Then, it should be noted that the GTB model trained on the strategy-politeness features and non-politeness features outperformed than the model on the non-politeness features when $N$ was 20,25 , and 30. This suggests that strategy-politeness features might help improve the prediction of students' problem-solving performance in the middle of the tutoring process.

To gain a better understanding of the predictive power of different types of features, we measured the contribution of features to the prediction by conducting the ablation test, which is described in Sec.3.6.4. As the instructional strategies were mostly employed in the middle of the tutoring process [27], we decided to investigate the ablation

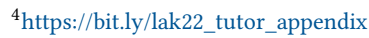


Table 4. The ablation test results of the GTB model when only considering the first 20 utterances in dialogue. Here, Row 1 of Table 4 presents the performance of the GTB model taking all strategy-politeness and non-politeness features as the input, and Rows 2-6 present the performance of the GTB model without taking a specific type of feature as input. The percentages in the brackets were calculated by taking the model performance of considering all features (i.e., Row 1 ) as the comparison. The results with the maximum performance decrease are in bold.

\begin{tabular}{lllll}
\hline Feature & Accuracy & F1 score & Cohen's k & AUC \\
\hline 1. Strategy + Non-politeness Feat. & 0.730 & 0.692 & 0.428 & 0.694 \\
\hline 2. w/o Non-politeness Feat. & $\mathbf{0 . 6 8 7}(-\mathbf{5 . 8 9 \%})$ & $\mathbf{0 . 6 2 3}(-\mathbf{9 . 9 7 \% )}$ & $\mathbf{0 . 2 9 2}(\mathbf{- 3 1 . 7 8 \% )}$ & $\mathbf{0 . 6 2 7}(-\mathbf{9 . 6 5 \%})$ \\
3. w/o Strategy-politeness Feat. & $0.722(-1.10 \%)$ & $0.682(-1.45 \%)$ & $0.413(-3.50 \%)$ & $0.689(-0.72 \%)$ \\
4. w/o Strategy-politeness Feat. (Mean) & $0.728(-0.27 \%)$ & $0.692(0.00 \%)$ & $0.427(-0.23 \%)$ & $0.694(0.00 \%)$ \\
5. w/o Strategy-politeness Feat. (Median) & $0.726(-0.55 \%)$ & $0.689(-0.43 \%)$ & $0.421(-1.64 \%)$ & $0.692(-0.29 \%)$ \\
6. w/o Strategy-politeness Feat. (Std.) & $0.723(-0.96 \%)$ & $0.684(-1.16 \%)$ & $0.413(-3.50 \%)$ & $0.687(-1.01 \%)$ \\
\hline
\end{tabular}

results when only considering the first 20 utterances as input. Here, instead of focusing on each feature, we were more interested in analysing the contributions made by each group of features. It should be noted that our previous study [21] had demonstrated contributions of non-politeness features. Hence, the current study did not expand the analysis on specific features in the non-politeness features. For the strategy-politeness features, not only do we present the feature importance of strategy-politeness features (i.e., Row 3, Table 4) but also break down the strategy-politeness features into three sub-groups of features (i.e., Row 4-6, Table 4) as described in Sec. 3.6.2. Though the non-politeness features contributed to the prediction performance the most, the strategy-politeness features also demonstrated the potentials to improve the model's performance in four metrics.

\section{DISCUSSION AND CONCLUSION}

The current study contributed with the following main findings that are of relevance to human-human online tutoring practice and the design of dialogue-based intelligent tutoring systems:

- Overall, tutors were inclined to use more direct expressions in the instructional strategies of comprehensivegauging questions and negative feedback and use less direct expressions in the positive feedback.

- In tutorial sessions where students solved problems, tutors tended to ask more polite open questions and deliver more direct corrective feedback (negative feedback and elaborated positive feedback) for the students without prior progress than the students with prior progress.

- By incorporating the features of instructional strategies politeness with the features proposed in [21] (e.g., the sentiment levels of the utterance, complexity, and informativeness), the GTB model achieved better model performance of predicting student performance compared to our previous work [21],

\subsection{Implications}

The current study investigated the correlation between politeness and instructional communication from real-world human-human online dialogue tutoring. Our study provided guidelines for human tutors to consider the extent to which politeness can be expressed in instructional communication. Firstly, the findings suggested that tutors could use direct expression for the strategies Evaluation Questions and Negative Feedback as long as the instruction that tutors deliver meets the students' needs of solving problems, which is in line with the claim by [8]. As human tutors were reluctant to impose unpleasant feelings on students [7], they often spent much time on the word formulation of some instructional communication (e.g., providing negative feedback and directive hints) in tutoring which might hinder the efficiency of the tutoring $[7,8]$. Our study found that the tutors commonly guided students in less polite expressions for the instructional strategies Evaluation Questions and Negative Feedback. The strategy Negative 
Feedback was used to help the students identify errors (e.g., "No, it is wrong"). It is inevitable to be direct when using this strategy in order to point out the students' errors even though this tone of expression might appear to neglect the students' expectation for the appreciation [8].

Secondly, the current study showed that tutors asked more polite open questions to guide students without prior progress than the ones with prior progress. The strategy Open Question was used to gauge students' understanding and progress on the problem (e.g., "Why do you use this formula?"). Such questions could elicit students' self-explanation (i.e., students explain their understanding of the discussed topics) which proven to have benefits for learning [2, 10]. It should be noted that students with low prior knowledge are not confident to demonstrate self-explanation activities [2] and they commonly engaged more with polite tutors [24,32]. As our study investigated the student prior progress as the proxy of student prior knowledge, we suggested that tutors should provide more polite open questions (e.g., "What do you think we could try next?") to the students without prior progress compared to the students with prior progress.

Thirdly, our results (Fig. 1) showed that most tutors delivered positive feedback in a non-direct form. The strategy Positive Feedback was used to verify the students' correctness and encourage them to make more effort on the problems. Providing positive feedback in a direct form (e.g, "Right, that's exactly what you should get") might mitigate the advantages of positive feedback in building a friendly relationship with students since students expected receiving the praise and approval from tutors when students made correct steps [40]. In the future, it is worth further investigating the relationship between the use of politeness in positive feedback and students' learning experience by designing randomised controlled experiments.

The results of the current study shed some light on the design of future dialogue-based intelligent tutoring system (ITS) about how to express politely in the instructional process. Most of the existing studies have only examined instructional politeness in a binary form (i.e., either polite or direct) $[23,24,26,36,38,39]$ but neglected that instructional communication, in real-world tutoring, could be expressed in different surface forms, which can represent different politeness levels [33]. Based on the results of the current study, we submit that dialogue-based ITSs should not only focus on a single form of polite or direct instruction. In particular, it is important to make the trade-off between polite and direct expression in the tutoring since the polite tutors are more approachable for students[6,8] and direct tutors made the tutoring sessions more efficient $[7,8,30]$. To this end, it was necessary to understand the politeness levels of instructional strategies. Our study demonstrated the politeness levels of instructional strategies by observing human expert instruction, which was a typical method to further develop the design of ITS [13].

Finally, our prediction results demonstrated that the strategy-politeness features had the potential to predict the student's performance in the middle of the tutoring. In particular, by incorporating the strategy-politeness features in our feature set, the GTB model obtained the improvement of model performance in four evaluation metrics (e.g., F1 score,the AUC, and Cohen's $\kappa$ ) when considering the first 20 utterances as input. The results indicated that the use of politeness in the instructional strategies might correlate with the students' performance. Therefore, we suggested that the politeness of instructional strategies should be incorporated in the future work of predicting student performance.

\subsection{Limitations}

We acknowledge there are several limitations in the current study. Firstly, our results were limited to the United States cultural background. The tutors in our dataset were trained to teach students politely followed the policy of the educational technology company based in US. However, the students from different cultural backgrounds (e.g., those Asian countries) might have different perceptions of politeness. It is necessary to conduct a study investigating the variance of politeness levels in tutoring communication from the perspective of other cultures. Then, we did not 
analyse politeness across different subject areas (e.g., maths, chemistry, and physics). Our dataset contained the maths tutoring in more than $92 \%$ of the tutoring sessions, so our results might demonstrate the patterns in relation to maths tutoring. In our future work, it is worth to collect more dialogue sessions in other subjects (e.g., physics and chemistry) and analyse these dialogues separately regarding the different subjects. Thirdly, our study only examined the politeness levels of instructional strategies from the tutors' perspective. It still remains unknown whether the students prefer collaborating with the tutor who expressed the politeness in the instructional communication based on our analysed results. To further guide the practice of tutoring and the development of dialogue-based ITS, a randomised experiment should be conducted to investigate the students' preference on the politeness of instructional communication. Lastly, since we measured students' prior progress from the first few utterances, it may happen that some students started talking about this progress outside of that window, i.e., after the utterances that have been taken for the analysis.

\subsection{Future Work}

Our study obtained the results by examining real-world human-human tutoring. The results demonstrated that most tutors expressed politely in some instructional strategies such as positive feedback. However, some tutors might deliver positive feedback in a direct form (e..g, "Right, that's what you should get"), which might lead to negative effects on tutoring. Therefore, we plan to construct a politeness transfer tool to automatically convert non-polite tutors' expressions into polite forms. Then, as indicated in Sec. 3.6.2, we did not incorporate students' prior progress in the feature set for student performance prediction. In the future work, we plan to build a machine learning model to automatically identify the students' prior progress in the tutoring process and include it as an additional feature for predicting student performance.

\section{REFERENCES}

[1] Iz Beltagy, Kyle Lo, and Arman Cohan. 2019. SciBERT: A Pretrained Language Model for Scientific Text. In Proceedings of the 2019 Conference on EMNLP-IFCNLP. 3606-3611.

[2] Kirsten Berthold, Tessa HS Eysink, and Alexander Renkl. 2009. Assisting self-explanation prompts are more effective than open prompts when learning with multiple representations. Instructional Science 37, 4 (2009), 345-363.

[3] Tasha Bleistein and Marilyn Lewis. 2014. One-on-one language teaching and learning: Theory and practice. Springer.

[4] Benjamin S Bloom. 1984. The 2 sigma problem: The search for methods of group instruction as effective as one-to-one tutoring. Educational researcher 13, 6 (1984), 4-16.

[5] Susan E Brennan and Herbert H Clark. 1996. Conceptual pacts and lexical choice in conversation. Journal of experimental psychology: Learning, memory, and cognition 22, 6 (1996), 1482.

[6] Penelope Brown and Stephen Levinson. 1987. Politeness: some universals in language usage. Cambridge University Press, Cambridge, UK.

[7] Benjamin Brummernhenrich and Regina Jucks. 2013. Managing face threats and instructions in online tutoring. Fournal of Educational Psychology 105, 2 (2013), 341.

[8] Benjamin Brummernhenrich and Regina Jucks. 2016. "He shouldn't have put it that way!" How face threats and mitigation strategies affect person perception in online tutoring. Communication Education 65, 3 (2016), 290-306.

[9] Tianqi Chen and Carlos Guestrin. 2016. Xgboost: A scalable tree boosting system. In Proceedings of the 2016 Conference on KDD. 785-794.

[10] Jennifer L Chiu and Michelene TH Chi. 2014. Supporting self-explanation in the classroom. Applying science of learning in education: Infusing psychological science into the curriculum (2014), 91-103.

[11] Kevyn Collins-Thompson. 2014. Computational assessment of text readability: A survey of current and future research. ITL-International fournal of Applied Linguistics 165, 2 (2014), 97-135.

[12] Thomas G Dietterich et al. 2002. Ensemble learning. The handbook of brain theory and neural networks 2 (2002), 110-125.

[13] Benedict Du Boulay and Rosemary Luckin. 2016. Modelling human teaching tactics and strategies for tutoring systems: 14 Years on. International Journal of Artificial Intelligence in Education 26, 1 (2016), 393-404.

[14] Judi Simmons Estes. 2017. Teacher preparation programs and learner-centered, technology-integrated instruction. In Handbook of research on learner-centered pedagogy in teacher education and professional development. IGI Global, 85-103.

[15] Manuel Fernández-Delgado, Eva Cernadas, Senén Barro, and Dinani Amorim. 2014. Do we need hundreds of classifiers to solve real world classification problems? The journal of machine learning research 15, 1 (2014), 3133-3181. 
[16] Clayton Hutto and Eric Gilbert. 2014. Vader: A parsimonious rule-based model for sentiment analysis of social media text. In Proceedings of the International AAAI Conference on Web and Social Media, Vol. 8.

[17] Samantha Jiménez, Reyes Juárez-Ramírez, Victor H Castillo, and Juan José Tapia Armenta. 2018. Affective Feedback in Intelligent Tutoring Systems: A Practical Approach. Springer.

[18] Regina Jucks, Lena Päuler, and Benjamin Brummernhenrich. 2016. "I need to be explicit: You're wrong”: Impact of face threats on social evaluations in online instructional communication. Interacting with Computers 28, 1 (2016), 73-84.

[19] Jionghao Lin, David Lang, Haoran Xie, Dragan Gašević, and Guanliang Chen. 2020. Investigating the Role of Politeness in Human-Human Online Tutoring. In Artificial Intelligence in Education. Springer International Publishing, Cham, 174-179.

[20] Jionghao Lin, Shirui Pan, Cheng Siong Lee, and Sharon Oviatt. 2019. An Explainable Deep Fusion Network for Affect Recognition Using Physiological Signals. In Proceedings of the 28th ACM International Conference on Information and Knowledge Management (Beijing, China) (CIKM '19). ACM, New York, NY, USA, 2069-2072.

[21] Jionghao Lin, Shaveen Singh, Lele Sha, Wei Tan, David Lang, Dragan Gašević, and Guanliang Chen. 2022. Is it a good move? Mining effective tutoring strategies from human-human tutorial dialogues. Future Generation Computer Systems 127 (2022), $194-207$.

[22] Richard E Mayer, W Lewis Johnson, Erin Shaw, and Sahiba Sandhu. 2006. Constructing computer-based tutors that are socially sensitive: Politeness in educational software. International fournal of Human-Computer Studies 64, 1 (2006), 36-42.

[23] Bruce M McLaren, Krista E DeLeeuw, and Richard E Mayer. 2011. Polite web-based intelligent tutors: Can they improve learning in classrooms? Computers \& Education 56, 3 (2011), 574-584.

[24] Bruce M McLaren, Krista E DeLeeuw, and Richard E Mayer. 2011. A politeness effect in learning with web-based intelligent tutors. International Journal of Human-Computer Studies 69, 1-2 (2011), 70-79.

[25] Bruce M McLaren, Sung-Joo Lim, David Yaron, and Kenneth R Koedinger. 2007. Can a polite intelligent tutoring system lead to improved learning outside of the lab? Frontiers in Artificial Intelligence and Applications 158 (2007), 433.

[26] Maria Mikheeva, Sascha Schneider, Maik Beege, and Günter Daniel Rey. 2019. Boundary conditions of the politeness effect in online mathematical learning. Computers in Human Behavior 92 (2019), 419-427.

[27] Donald M Morrison, Benjamin Nye, Vasile Rus, Sarah Snyder, Jennifer Boller, and Kenneth Miller. 2015. Tutorial dialogue modes in a large corpus of online tutoring transcripts. In International Conference on Artificial Intelligence in Education. Springer, 722-725.

[28] Kimberly A Neuendorf. 2017. The Content Analysis Guidebook. SAGE Publications.

[29] Tong Niu and Mohit Bansal. 2018. Polite dialogue generation without parallel data. Transactions of the ACL 6 (2018), $373-389$.

[30] Natalie K Pearson, Roger J Kreuz, Rolf A Zwaan, and Arthur C Graesser. 1995. Pragmatics and pedagogy: Conversational rules and politeness strategies may inhibit effective tutoring. Cognition and instruction 13, 2 (1995), 161-188.

[31] Edd Pitt and Lin Norton. 2017. 'Now that's the feedback I want!'Students' reactions to feedback on graded work and what they do with it. Assessment \& Evaluation in Higher Education 42, 4 (2017), 499-516.

[32] Kaśka Porayska-Pomsta, Manolis Mavrikis, and Helen Pain. 2008. Diagnosing and acting on student affect: the tutor's perspective. User Modeling and User-Adapted Interaction 18, 1-2 (2008), 125-173.

[33] Kaśka Porayska-Pomsta and Chris Mellish. 2004. Modelling politeness in natural language generation. In International Conference on Natural Language Generation. Springer, 141-150.

[34] Kaska Porayska-Pomsta and Helen Pain. 2004. Providing cognitive and affective scaffolding through teaching strategies: applying linguistic politeness to the educational context. In International conference on intelligent tutoring systems. Springer, 77-86.

[35] Vasile Rus, Nabin Maharjan, Lasang Jimba Tamang, Michael Yudelson, Susan Berman, Stephen E Fancsali, and Steve Ritter. 2017. An analysis of human tutors' actions in tutorial dialogues. In The Thirtieth International Flairs Conference.

[36] Sascha Schneider, Steve Nebel, Simon Pradel, and Günter Daniel Rey. 2015. Mind your Ps and Qs! How polite instructions affect learning with multimedia. Computers in Human Behavior 51 (2015), 546-555.

[37] Alexandria Katarina Vail and Kristy Elizabeth Boyer. 2014. Identifying Effective Moves in Tutoring: On the Refinement of Dialogue Act Annotation Schemes. In Intelligent Tutoring Systems.

[38] Ning Wang and W Lewis Johnson. 2008. The Politeness Effect in an intelligent foreign language tutoring system. In ITS. Springer, 270-280.

[39] Ning Wang, W Lewis Johnson, Richard E Mayer, Paola Rizzo, Erin Shaw, and Heather Collins. 2008. The politeness effect: Pedagogical agents and learning outcomes. International journal of human-computer studies 66, 2 (2008), 98-112.

[40] Ning Wang, W Lewis Johnson, Paola Rizzo, Erin Shaw, and Richard E Mayer. 2005. Experimental evaluation of polite interaction tactics for pedagogical agents. In Proceedings of the 10th international conference on Intelligent user interfaces. 12-19.

[41] Qi Wang and Liping Shen. 2018. Student proficiency prediction on CNMOOC data. In Proceedings of the 2nd International Conference on Machine Learning and Soft Computing. 15-18.

[42] Fan Yang and Frederick WB Li. 2018. Study on student performance estimation, student progress analysis, and student potential prediction based on data mining. Computers \& Education 123 (2018), 97-108. 